\title{
Avaliação da usabilidade de hipermídia para ensino da sistematização da assistência de enfermagem
}

\author{
Pétala Tuani Candido de Oliveira Salvador \\ Professora da Escola de Saúde da Universidade Federal do Rio Grande do Norte. Natal, \\ Rio Grande do Norte, Brasil \\ $\triangle$ petalatuani@hotmail.com
}

\section{Flávia Barreto Tavares Chiavone}

Doutoranda em Enfermagem pelo Programa de Pós-Graduação em Enfermagem da Universidade Federal do Rio Grande do Norte. Natal, Rio Grande do Norte, Brasil

\section{Eliana Amaral}

Professora-Titular da Universidade Estadual de Campinas. Campinas, São Paulo, Brasil

\section{Maria Isabel Domingues Fernandes}

Professora Adjunta na Unidade Científico-Pedagógica de Enfermagem Médico-Cirúrgica da Escola Superior de Enfermagem de Coimbra. Coimbra, Portugal

\section{Viviane Euzébia Pereira Santos}

Professora do Departamento de Enfermagem e do Programa de Pós-Graduação em Enfermagem da Universidade Federal do Rio Grande do Norte. Natal, Rio Grande do Norte, Brasil

\section{Resumo:}

o processo de avaliação de uma tecnologia educacional objetiva analisar o grau em que o recurso se mostra apropriado para alcançar os seus objetivos, a partir de uma análise sistemática por sujeitos de diferentes grupos, a depender do aspecto que será avaliado. Neste estudo, destaca-se o processo de avaliação de usabilidade de uma hipermídia para apoiar o ensino da sistematização da assistência de enfermagem para discentes dos cursos técnico e de graduação em enfermagem. Objetiva-se avaliar a usabilidade de hipermídia para apoio ao ensino da sistematização da assistência de enfermagem. Estudo descritivo, com abordagem quantitativa. Foi aplicado um questionário eletrônico composto por questões de caracterização, uma adaptação da System Usability Scale e do Suitability Assessment of Materials. A coleta de dados foi realizada de novembro de 2017 a janeiro de 2018, com nove docentes e 11 discentes dos Cursos Superior e Técnico em Enfermagem de uma universidade pública do Nordeste do Brasil. Os dados foram analisados por estatística descritiva. O Score System Usability Scale total foi de 91,9 (docentes) e 83,9 (discentes). Docentes e discentes consideraram a hipermídia eficiente, facilitadora de aprendizagem e demonstraram satisfação com sua usabilidade. A hipermídia foi avaliada com boa e excelente usabilidade pelos discentes e docentes, respectivamente. A hipermídia, disponibilizada no link http://escoladesaude.ufrn.br/saeufrn/, pode ser utilizado pelas diversas instituições de ensino que formam profissionais de enfermagem dos diferentes níveis acadêmicos, bem como pelos serviços assistenciais, como um subsídio para a efetivação desse campo de saber.

Palavras-chave: Tecnologia educacional, materiais de ensino, processos de enfermagem, educação em enfermagem, docentes de enfermagem. 


\title{
Evaluation of hypermedia usability for teaching nursing care systematization
}

\begin{abstract}
:
The process of evaluating an educational technology aims to analyze the degree to which the resource is appropriate to achieve its objectives, based on a systematic analysis by subjects of different groups, depending on the aspect that will be evaluated. In this study, we highlight the process of usability evaluation of a hypermedia to support the teaching of the systematization of nursing care for students of technical and undergraduate nursing courses. The objective is to evaluate the usability of a hypermedia to support the teaching of nursing care systematization. Descriptive study with quantitative approach. An electronic questionnaire consisting of characterization questions, an adaptation of the System Usability Scale and the Suitability Assessment of Materials was applied. Data collection was performed from November 2017 to January 2018, with nine teachers and 11 students from the Higher and Technical Nursing Courses of a public university in Northeastern Brazil. Data were analyzed by descriptive statistics. The total Score System Usability Scale was 91.9 (teachers) and 83.9 (students). Teachers and students considered the hypermedia efficient, facilitating learning and demonstrated satisfaction with its usability. The hypermedia was evaluated with good and excellent usability by students and teachers. Hypermedia, available in the link http://escoladesaude.ufrn.br/saeufrn/, can be used by the various educational institutions that train nursing professionals of different academic levels, as well as by care services, as a subsidy for the effectiveness of this field of knowledge.
\end{abstract}

Keywords: Educational technology, teaching materials, nursing process, education nursing, faculty nursing.

\section{Evaluación de la usabilidad de hipermedia para la enseñanza de sistematización de cuidados de enfermería}

\section{Resumen:}

El proceso de evaluación de una tecnología educativa tiene como objetivo analizar el grado en que el recurso es adecuado para alcanzar sus objetivos, a partir de un análisis sistemático por parte de sujetos de diferentes grupos, dependiendo del aspecto que se evalúe. En este estudio, destacamos el proceso de evaluación de usabilidad de un hipermedia para apoyar la enseñanza de la sistematización de la atención de enfermería para estudiantes de cursos técnicos y enfermería de grado. El objetivo es evaluar la usabilidad de una hipermedia para apoyar la enseñanza de la sistematización del cuidado de enfermería. Estudio descriptivo con enfoque cuantitativo. Se aplicó un cuestionario electrónico que consta de preguntas de caracterización, una adaptación de la System Usability Scale y de la Suitability Assessment of Materials. La recolección de datos se realizó de noviembre de 2017 a enero de 2018, con nueve maestros y 11 estudiantes de los cursos de enfermería superior y técnica de una universidad pública en el noreste de Brasil. Los datos fueron analizados por estadística descriptiva. El Score System Usability Scale fue 91.9 (docentes) y 83.9 (estudiantes). Los profesores y los estudiantes consideraron la hipermedia eficiente, facilitando el aprendizaje y demostrando satisfacción con su usabilidad. La hipermedia fue evaluado con buena y excelente usabilidad por estudiantes y maestros. La hipermedia disponible en el enlace http://escoladesaude.ufrn.br/saeufrn/ puede ser utilizados por las diversas instituciones educativas que forman profesionales de enfermería de diferentes niveles académicos, así como por los servicios de cuidado, como un subsidio para la efectividad de este campo del conocimiento.

Palabras clave: Tecnología educacional, materiales de enseñanza, proceso de enfermería, educación en enfermería, docentes de enfermería.

\section{INTRODUÇÃO}

Contemporaneamente, vivenciam-se mudanças nos processos educacionais, em parte decorrentes do surgimento de uma geração de alunos conhecida como Net Generation ou estudantes 3.0, inseridos e influenciados por um ambiente repleto de mídias e multitarefas (GOÉS et al., 2015). 
O uso de recursos digitais cresceu rapidamente nas últimas décadas e o ensino através de tecnologias foi amplamente adotado nos diferentes níveis educacionais, tanto em ambientes presenciais como de e-learning (ÁLVAREZ-NIETO et al., 2018; BUTTON; HARRINGTON; BELAN, 2014).

Processo semelhante é visualizado no ensino em enfermagem, em que se observa um aumento da utilização de tecnologias como mecanismos didáticos capazes de promover interatividade e incidir, inclusive, no aumento da segurança no processo decisório do cuidado (PEREIRA et al., 2016; FROTA et al., 2018).

Ganha destaque, nesse contexto, o uso de hipermídias, que consistem em recursos digitais de aprendizagem flexíveis e interativos, que proporcionam ao discente a oportunidade de explorar o conteúdo de acordo com suas necessidades e a partir de múltiplas mídias (FROTA et al., 2018).

De forma preocupante, porém, é relatada na literatura a escassez de publicações sobre hipermídias e seu uso adequado na educação em enfermagem, sobretudo no que concerne ao ensino técnico desta profissão (GOÉS et al., 2015; SPERONI; VIZZOTTI, 2015).

Relata-se, ainda, que por vezes a seleção dos recursos tecnológicos pelos docentes ocorre com base na facilidade de acesso, ao invés da capacidade de atingir os objetivos educacionais adequados, o que pode comprometer a qualidade e prejudicar o sucesso da aprendizagem (GARCÍA-BARRERA, 2016).

Nesse sentido, é imperativo que as tecnologias educacionais sejam desenvolvidas e avaliadas por meio de indicadores de credibilidade que permitam certificar sua qualidade e utilidade educacional (ÁLVAREZ-NIETO et al., 2018).

O processo de avaliação de uma tecnologia educacional objetiva analisar o grau em que o recurso se mostra apropriado para alcançar os seus objetivos, a partir de uma análise sistemática por sujeitos de diferentes grupos, a depender do aspecto que será avaliado (TEIXEIRA; MOTA, 2011; PASQUALI, 2010).

Neste estudo, destaca-se o processo de avaliação de usabilidade de uma hipermídia, que se configura como recurso digital de tamanho limitado e que pode ser reutilizado dentro de várias atividades e estratégias pedagógicas (SALVADOR et al., 2017). 
A hipermídia foi desenvolvida para apoiar o ensino da Sistematização da Assistência de Enfermagem (SAE) para discentes dos cursos técnico e de graduação em enfermagem (SALVADOR et al., 2018). A SAE se configura enquanto instrumento metodológico que orienta o cuidado profissional da Enfermagem e a documentação da prática profissional, sendo reconhecido, deste 2009, como estratégia essencial a ser utilizada pela equipe de enfermagem para qualificar o seu cuidado (SALVADOR et al., 2017).

Esta hipermídia passou por um processo de validação de conteúdo, que foi realizado com experts da enfermagem e revelou um Coeficiente de Validade de Conteúdo (CVC) de 0,96 (SALVADOR et al., 2018). Após isto, emergiu a necessidade de se avaliar a usabilidade da hipermídia pelo público que irá utilizá-la, quais sejam: docentes e discentes de enfermagem.

A usabilidade é um indicador de qualidade que mede o quão fácil de usar alguma coisa é. De acordo com a International Organization for Standardization (ISO) 9241, a avaliação da usabilidade deve integrar a análise da eficiência (realização da tarefa sem erros), eficácia (realização da tarefa com otimização dos recursos disponíveis) e satisfação (o quanto as expectativas são alcançadas) (ABNT, 2011).

Ao apresentar o processo de avaliação da usabilidade de uma hipermídia para apoiar o ensino de enfermagem, busca-se incentivar a reflexão sobre a importância de se desenvolver, validar e avaliar tecnologias educacionais a partir de referenciais pedagógicos e metodológicos adequados.

Ao mesmo tempo em que se espera contribuir com a descrição de referencial metodológico de avaliação de usabilidade de hipermídia, apresenta-se ao final do manuscrito o seu link de acesso, que pode ser utilizado nos diferentes espaços de educação em enfermagem, com o potencial de disseminação de conhecimentos acerca da SAE, tema transversal a ser trabalhado em todos os níveis educacionais da profissão.

Apresenta-se, desse modo, como questão norteadora da presente pesquisa: como docentes e discentes avaliam a usabilidade de hipermídia para apoiar o ensino da sistematização da assistência de enfermagem?

Objetiva-se, portanto, avaliar a usabilidade de hipermídia para apoio ao ensino da sistematização da assistência de enfermagem. 


\section{MATERIAL E MÉTODOS}

Estudo descritivo, de abordagem quantitativa, norteado pelo referencial teórico metodológico da psicometria (PASQUALI, 2010). Constitui etapa final de pesquisa metodológica que buscou construir, validar e avaliar hipermídia para apoio ao ensino da sistematização da assistência de enfermagem.

A população da pesquisa foi composta por docentes e discentes dos Cursos Superior e Técnico em Enfermagem de uma universidade pública do Nordeste do Brasil.

Foram convidados a participar docentes efetivos que atuavam em unidades curriculares que integram o ensino da SAE. Para os discentes, foi estabelecido como critério de inclusão ser aluno do último período do curso correspondente. Excluíram-se docentes e discentes que não completaram o envio do formulário eletrônico.

Referenciais teóricos de processos de avaliação de instrumentos não estabelecem um número ideal de sujeitos, mas orientam um número de seis a vinte participantes (PASQUALI, 2010). Além disso, pesquisas que utilizam formulários eletrônicos relatam um significativo número de perdas, de modo que são esperadas taxas de resposta em torno de 15\% (GOÉS et al., 2015; SALVADOR et al., 2018).

Com base nos relatos das altas taxas de não resposta de formulários eletrônicos, optou-se por não realizar um cálculo amostral, de modo que se convidou para participar da pesquisa todos os docentes e discentes componentes da população do estudo.

Desse modo, 12 docentes e 95 discentes receberam convite via e-mail para avaliar o OVA e nove docentes e 11 discentes responderam o questionário eletrônico (taxa de resposta de 75,0\% e $11,6 \%$, respectivamente) (tabela 1 ).

Tabela 1 - Taxa de resposta do formulário eletrônico

\begin{tabular}{ccccccc}
\hline \multirow{2}{*}{ Curso } & \multicolumn{4}{c}{ Docentes } & \multicolumn{3}{c}{ Discentes } \\
\cline { 2 - 7 } & $\mathbf{N}$ & $\mathbf{n}$ & Taxa de Resposta (\%) & $\mathbf{N}$ & $\mathbf{n}$ & Taxa de Resposta (\%) \\
\hline Técnico & 6 & 5 & 83,3 & 40 & 7 & 17,5 \\
Graduação & 6 & 4 & 66,7 & 55 & 4 & 7,3 \\
\hline Total & $\mathbf{1 2}$ & $\mathbf{9}$ & $\mathbf{7 5 , 0}$ & $\mathbf{9 5}$ & $\mathbf{1 1}$ & $\mathbf{1 1 , 6}$ \\
\hline
\end{tabular}

Fonte: Dados da pesquisa, 2018. 
A coleta de dados foi realizada de novembro de 2017 a janeiro de 2018. Os docentes e os discentes selecionados receberam um convite via e-mail para participação. Foram instruídos a navegar pela hipermídia e então avaliar sua usabilidade. Para isto, eles responderam um formulário eletrônico construído a partir do Google Forms composto por três partes: 1) questionário de caracterização; 2) adaptação da System Usability Scale (SUS); e 3) adaptação do Suitability Assessment of Materials (SAM).

Para os docentes, o questionário de caracterização foi composto por perguntas relativas à idade, ao sexo, à maior titulação acadêmica, ao tempo de experiência na docência e à experiência no uso de tecnologia educacional. Aos discentes foi questionado idade, sexo e curso.

Quanto à avaliação da usabilidade da hipermídia, para as duas populações de estudo foi utilizada uma adaptação da SUS, a mais utilizada e reconhecida mundialmente para avaliação deste indicador. Desenvolvido por John Brooke, em 1986, é considerado um instrumento robusto, de domínio público, sendo composto por 10 questões objetivas que utilizam uma escala Likert de 5 pontos, onde 0 equivale a "discordo plenamente" e 5 a “concordo plenamente" (BANGOR; KORTUM; MILLER, 2009).

Por se tratar de um material educacional, a avaliação da usabilidade, sobretudo no que concerne ao princípio da satisfação, foi acrescida de uma adaptação do SAM, cuja autorização de uso foi concedida pelas pesquisadoras que o traduziram para o português. O SAM tem por objetivo avaliar a dificuldade e conveniência dos materiais educativos quanto a sua satisfação de uso, utilizando uma escala de resposta tipo Likert (0=inadequado, 1=parcialmente adequado, 2=adequado) para atributos relacionados a conteúdo, linguagem, ilustrações, layout, motivação e aspectos culturais (SOUSA; TURRINI; POVEDA, 2015).

Após o envio do e-mail convite aos participantes, foi enviado três lembretes de participação na pesquisa, a cada 15 dias. Após este processo, a coleta de dados foi considerada encerrada. Apesar da alta taxa de não resposta dos discentes, como o número absoluto de sujeitos respondentes estava dentre do recomendado pelo referencial teórico utilizado (de seis a vinte participantes) (PASQUALI, 2010), optou-se por não realizar uma nova rodada de envio do formulário após se completar o envio dos três lembretes. 
As variáveis de caracterização dos sujeitos de pesquisa foram analisadas a partir de estatística descritiva simples.

Para avaliar a usabilidade da hipermídia, foi calculado o Score SUS Total. O resultado obtido em uma aplicação da SUS se dá a partir da soma das pontuações de cada questão: para as questões ímpares a contribuição é calculada pela posição da escala menos 1; e para as questões pares, calcula-se 5 menos o valor da posição da escala. Multiplica-se a soma dos valores por 2,5 e obtém-se o Score SUS Total, cuja amplitude varia de 0 a 100. Para classificação do Score obtido, utilizaram-se os parâmetros: <51 é considerado ruim; >71 é avaliado como bom; >86 é excelente; e >91 é a melhor usabilidade alcançável. Foi estabelecido o ponto de corte do Score SUS Total de 70 para considerar a hipermídia válida em usabilidade (BANGOR; KORTUM; MILLER, 2009).

Foi avaliado, ainda, o Score SUS dos componentes de qualidade de usabilidade a partir do agrupamento das questões da SUS: facilidade de aprendizagem (questões 3, 4, 7 e 10); eficiência (questões 5, 6 e 8); facilidade de memorização (questão 2); minimização dos erros (questão 6); e satisfação (questões 1, 4 e 9) (BANGOR; KORTUM; MILLER, 2009).

Quanto à avaliação dos itens do SAM, foi utilizada a técnica Delphi, a qual consiste na avaliação de um instrumento pelo grupo respondente a partir da análise da concordância dos mesmos (SOUSA; TURRINI; POOVEDA, 2015). Esta técnica ocorre de forma sistematizada e é executada quantas vezes forem necessárias, de modo que o processo de rodadas se repita até o alcance do consenso de 70\% a 80\% (PASQUALI, 2010).

Seguiram-se os preceitos éticos estabelecidos pela Resolução nº 466/2012, do Conselho Nacional de Saúde. A proposta do estudo foi apreciada e aprovada pelo Comitê de Ética e Pesquisa, por meio do Parecer Consubstanciado $n^{-}$2.142.586, de 28/06/2017, CAAE $n^{\circ}$ 67241217.0.3001.5568.

\section{RESULTADOS}

Nove docentes completaram o processo de avaliação da hipermídia, uma taxa de resposta de 75,0\%. Com média de idade de 39,2 anos ( $\mathrm{DP}=8,1$ ), sendo mínimo de 32 e máximo 
de 54 anos, predominaram docentes mulheres $(8 ; 88,9 \%)$ e com doutorado $(8 ; 88,9 \%)$ como maior habilitação académica. Os participantes eram experientes na docência, com uma média de 11,6 anos (DP = 4,6), mínimo de 7 e máximo de 20 anos. A maioria relatou ter experiência no uso de tecnologia educacional $(8 ; 88,9 \%)$ e referiu já terem utilizado em sala de aula: vídeos, jogos educativos, Kahoot, infográfico, aplicativos, Prezi, Mendley, Wiki, projetor multimídia, sites, Power Point e filmes.

Quanto à avaliação da usabilidade da hipermídia, os docentes consideraram a hipermídia válida, com Score SUS para todos os itens superior a 70,0 (gráfico 1).

Gráfico 1 - Score das respostas dos docentes aos itens da System Usability Scale (SUS)

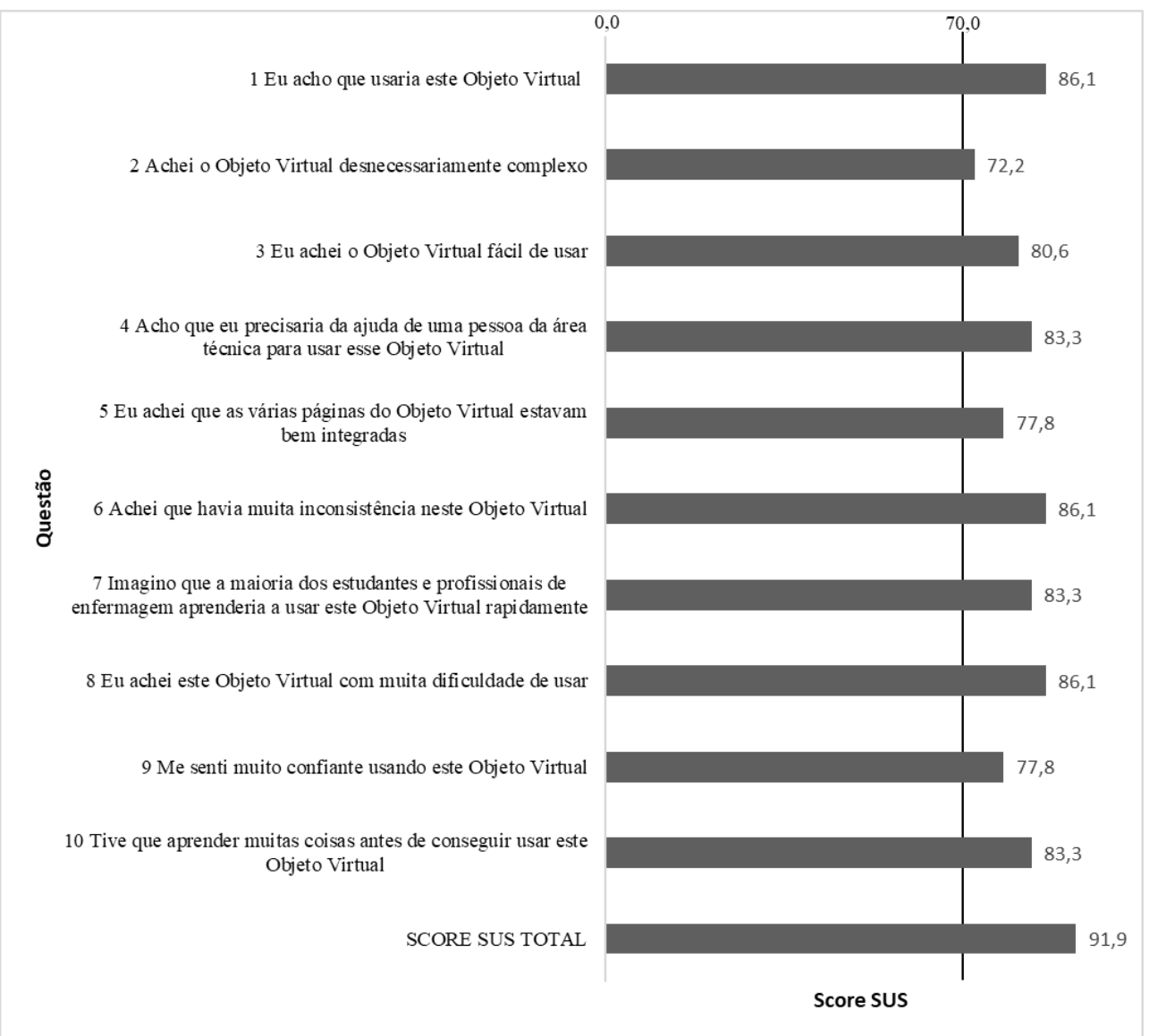

Fonte: Própria.

Assim, o Score SUS total obtido a partir da avaliação dos docentes foi de 91,9 (DP = 9,3), o que corresponde a uma excelente usabilidade. Os componentes de qualidade da usabilidade 
também obtiveram avaliações boas e excelentes pelos docentes: facilidade de aprendizagem - Score SUS de 82,6; eficiência - 83,3; facilidade de memorização - 72,2; minimização dos erros - 86,1; e satisfação - 82,4.

Todos os itens do SAM foram analisados como adequados por mais de 70,0\% dos docentes, o que revelou satisfação com o uso da hipermídia em todos os seus aspectos (tabela 2).

Tabela 2 - Respostas dos docentes aos itens adaptados do SAM*

\begin{tabular}{|c|c|c|c|}
\hline \multirow{2}{*}{ Item } & Inadequado & $\begin{array}{c}\text { Parcialmente } \\
\text { Adequado }\end{array}$ & Adequado \\
\hline & $\mathrm{n}(\%)$ & $\mathrm{n}(\%)$ & $\mathrm{n}(\%)$ \\
\hline \multicolumn{4}{|l|}{1 Conteúdo } \\
\hline $\begin{array}{l}1.1 \text { O conteúdo atende aos objetivos da } \\
\text { hipermídia }\end{array}$ & - & - & $9(100,0)$ \\
\hline $\begin{array}{l}\text { 1.2. As páginas da hipermídia são divididas } \\
\text { de forma coerente }\end{array}$ & - & - & $9(100,0)$ \\
\hline $\begin{array}{l}1.3 \text { O conteúdo atende às necessidades do } \\
\text { público alvo }\end{array}$ & - & $1(11,1)$ & $8(88,9)$ \\
\hline $\begin{array}{l}\text { 1.4 Existe lógica na sequência das páginas } \\
\text { da hipermídia }\end{array}$ & - & - & $9(100,0)$ \\
\hline $\begin{array}{l}1.5 \text { O conteúdo é relevante para ser } \\
\text { informado ao público alvo }\end{array}$ & - & - & $9(100,0)$ \\
\hline $\begin{array}{l}1.6 \text { o conteúdo está correto do ponto de } \\
\text { vista científico }\end{array}$ & - & - & $9(100,0)$ \\
\hline \multicolumn{4}{|l|}{ 2. Linguagem } \\
\hline $\begin{array}{l}2.1 \text { A redação é compatível com o público } \\
\text { alvo }\end{array}$ & - & - & $9(100,0)$ \\
\hline 2.2 As frases são atrativas e não cansativas & - & $1(11,1)$ & $8(88,9)$ \\
\hline $\begin{array}{l}\text { 2.3. Existem clareza e objetividade no } \\
\text { texto }\end{array}$ & - & - & $9(100,0)$ \\
\hline 3. Ilustrações & & & \\
\hline
\end{tabular}


3.1 Os elementos gráficos condizem com o conteúdo

$1(11,1) \quad 8(88,9)$

3.2 Os elementos gráficos são compreensíveis

$1(11,1)$

$8(88,9)$

3.3 O número de elementos gráficos é suficiente para abordar o conteúdo

$1(11,1)$

$8(88,9)$

4. Layout

4.1 O tamanho e fonte da letra favorece a leitura

4.2 As cores utilizadas na hipermídia viabilizam a leitura

4.3 A disposição dos itens e das páginas é organizada

$4.4 \mathrm{O}$ número de páginas e o tamanho do material é coerente

5. Motivação

5.1 O leitor é incentivado a prosseguir a leitura pelo conteúdo

5.2 A hipermídia é esclarecedora

$(11,1)$

$8(88,9)$

$1(11,1)$

$8(88,9)$

6. Cultura

6.1 A hipermídia atende aos vários perfis de estudantes/profissionais de enfermagem

$7(77,8)$

$1(11,1)$

$8(88,9)$

6.20 material é culturalmente adequado à linguagem e experiência do público alvo

6.3 Apresenta imagens e exemplos adequados culturalmente

$\begin{array}{lll}- & 2(22,2) & 7(77,8) \\ - & 1(11,1) & 8(88,9)\end{array}$

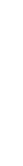

$9(100,0)$

*SAM - Suitability Assessment of Materials

Fonte: Dados da pesquisa, 2018.

Quanto aos discentes, a amostra final foi de 11 sujeitos respondentes, o que correspondeu a taxa de resposta de 11,6\%. A média de idade foi de 25,3 anos (DP = 5,5), mínimo de 19 e máximo de 39 anos. Houve predomínio das discentes mulheres (10; 90,9\%). 
A usabilidade da hipermídia também obteve boa avaliação pelos discentes, de modo que as respostas dos discentes denotaram um Score SUS para todos os itens superior a 70,0 (gráfico 2).

O Score SUS total obtido a partir da avaliação dos discentes foi de 83,9 (DP = 8,0), o que denota uma boa usabilidade. Os componentes de qualidade da usabilidade receberam avaliações boas e excelentes: facilidade de aprendizagem - Score SUS de 81,3; eficiência - 88,0; facilidade de memorização - 75,0; minimização dos erros - 83,3; e satisfação - 82,4.

Gráfico 2 - Score das respostas dos discentes aos itens da System Usability Scale (SUS)

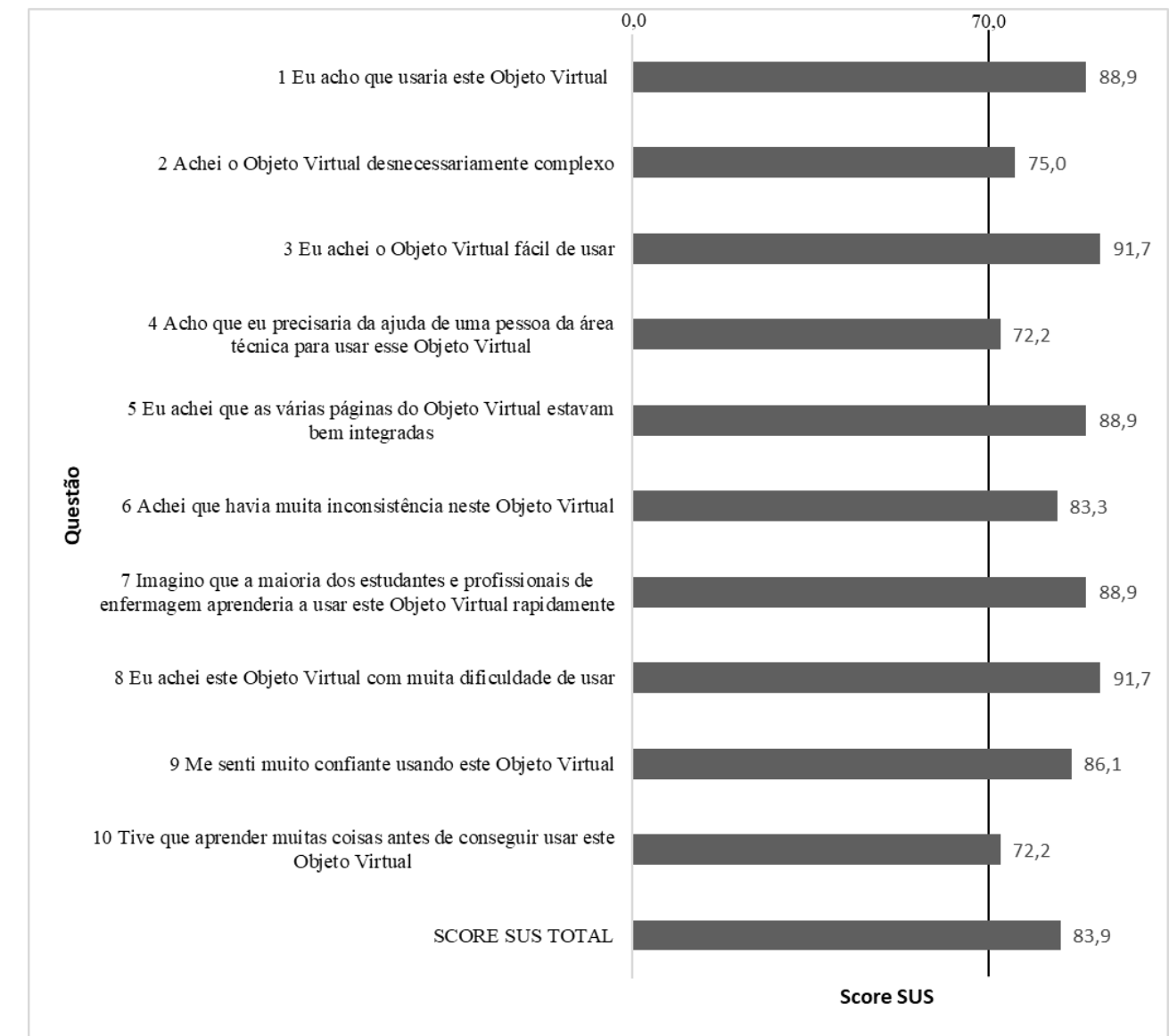

Fonte: Própria.

A satisfação com o uso da hipermídia também foi obtida com os discentes, com avaliação dos itens do SAM com mais de 70,0\% (tabela 3). 
Tabela 3 - Respostas dos discentes aos itens adaptados do SAM*

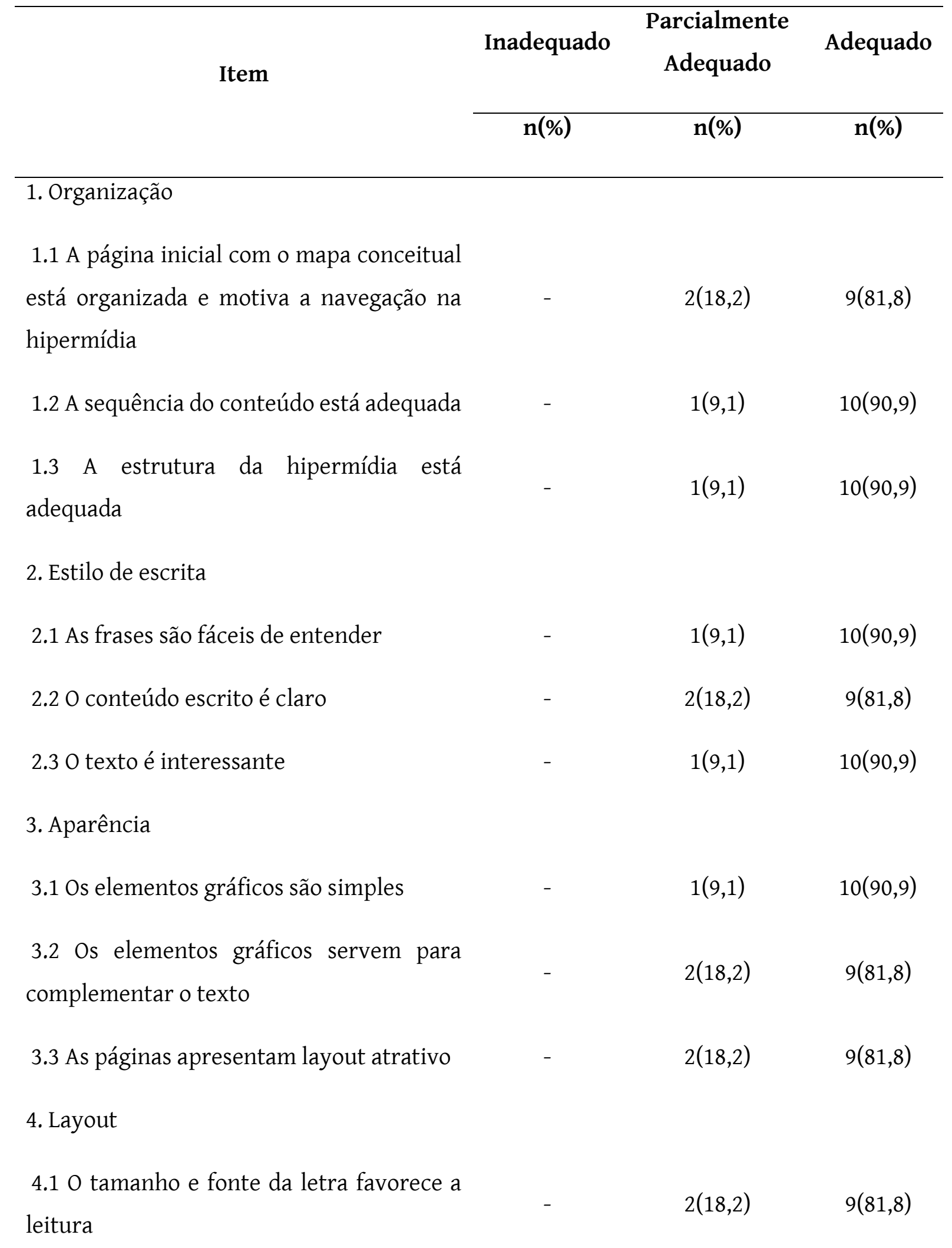


4.2 As cores utilizadas na hipermídia viabilizam a leitura

4.3 A disposição dos itens e das páginas é organizada

$1(9,1) \quad 10(90,9)$

4.4 O número de páginas e o tamanho do material é coerente

$3(27,3) \quad 8(72,7)$

5. Motivação

5.1 Em sua opinião, qualquer estudante/profissional de enfermagem que $1(9,1) \quad 10(90,9)$ acessar essa hipermídia vai entender

5.2 Você se sentiu motivado a acessar toda a hipermídia

*SAM - Suitability Assessment of Materials

Fonte: Dados da pesquisa, 2018.

\section{DISCUSSÃO}

A hipermídia foi considerada com excelente e boa usabilidade pelos docentes e discentes, respectivamente. Discentes e docentes consideraram a hipermídia eficiente, facilitadora da aprendizagem e demonstraram satisfação com a sua usabilidade.

A significativa taxa de não resposta ao formulário eletrônico dos discentes está de acordo com resultados de outros pesquisadores que utilizaram método de coleta de dados semelhantes (GOÉS et al., 2015; SALVADOR et al., 2018; COCCE et al., 2017). Para este público, sugere-se que outras técnicas de pesquisa podem ser mais eficientes, sobretudo as presenciais.

Em contrapartida, obteve-se uma alta taxa de resposta dos docentes, aspecto que pode ter relação com o perfil dos respondentes desta pesquisa, que indicaram, em sua maioria, ter experiência no uso de tecnologias educacionais. Trata-se, portanto, de um público que parece 
estar em preparado e motivado para os novos desafios do campo educacional (AGAMASARABIA et al., 2017).

Quanto aos dados de caracterização dos docentes e discentes participantes do estudo, denota-se que estes estão em coerência com os resultados da Pesquisa Perfil da Enfermagem no Brasil, com predominância nos dois públicos do sexo feminino (BRASIL, 2016).

Avaliou-se, nesta pesquisa, a usabilidade de uma hipermídia. Importante destacar, neste sentido, que pesquisadores compreendem que quando se avaliam tecnologias educacionais, é importante considerar três características básicas: qualidade ergonômica, técnica e estética (GOÉS et al., 2015; BRAGA et al., 2016). A qualidade técnica e estética da hipermídia recebeu avaliação satisfatória dos discentes e dos docentes, de modo que todos os itens do SAM receberam análise de sua adequabilidade superior a 70,0\%.

Enfatiza-se, nesse ínterim, a relevância de se considerar fundamentalmente os aspectos relacionados à aparência de hipermídia educacional, o que inclui pensar, desde o seu desenvolvimento, em aspectos referentes à linguagem, às ilustrações e ao layout, buscando promover um ambiente de aprendizagem interativo e motivador (BRAGA et al., 2016).

Como princípios, a linguagem da tecnologia educacional deve ser simples, objetiva, personalizada, instigante, acolhedora e convidativa. Deve ser mais coloquial, de modo a simular a oralidade e ser dialógica, pessoal, cordial, referenciada e adequada aos objetivos de aprendizagem, ao layout e aos tipos de materiais utilizados (HORTENSE; BERGEROT; DE DOMENICO, 2018). A linguagem e os demais recursos (ilustrações e outras mídias) devem buscar equilibrar a formalidade e informalidade, encorajar e favorecer a compreensão e realização das tarefas, com aproximações ao cotidiano do educando.

Numa perspectiva pedagógica de autonomia do discente, a hipermídia deve sempre focar neste sujeito e, por isso, deve favorecer a aproximação e o diálogo (BRAGA et al., 2016). Com a clareza e simplicidade da interface, facilita-se a identificação dos elementos mais importantes do aprendizado, de modo que os conteúdos são selecionados e abordados com objetividade e maiores chances de promover um aprendizado efetivo e significativo (HORTENSE; BERGEROT; DE DOMENICO, 2018). Na hipermídia avaliada, a interface é norteada pelos princípios da Teoria da Aprendizagem Significativa, com uma linguagem que procura 
dialogar com os discentes e promover um resgate de seus conhecimentos prévios (AUSUBEL, 2002).

O layout também busca favorecer a simplicidade da hipermídia, com um mapa conceitual que ilustra a conexão entre os conteúdos. A hipermídia incorpora, ainda, em suas telas, diferentes mídias, com o objetivo de motivar os diferentes sentidos e perfis de aprendizagem dos discentes, visto que as novas gerações de alunos nascem e crescem em um ambiente "telematizado", com gostos, atitudes e emoções diferenciados e influenciados por um ambiente com múltiplas mídias (AGAMA-SARABIA et al., 2017).

A qualidade ergonômica da hipermídia analisada, por sua vez, também obteve excelente avaliação, a partir do instrumento que buscou mensurar a sua usabilidade, a qual é compreendida como resultante da tríade efetividade, eficiência e satisfação do usuário (GOÉS et al., 2015; ABNT, 2011). Docentes e discentes avaliaram os critérios facilidade de aprendizagem, eficiência, satisfação e facilidade de memorização com bons índices na escala do Score SUS; e a capacidade de minimização de erros, ou seja, de clareza da proposta e da linguagem, com score excelente.

Em suma, conclui-se que a hipermídia foi considerada com boa usabilidade para apoiar o ensino da sistematização da assistência de enfermagem. Enfatiza-se, porém, que seu uso é incentivado na perspectiva de apoio ao ensino presencial, o que requer uma abordagem pedagógica do docente como fundamental. É indispensável afirmar que as tecnologias não dispensam o docente. São uma ferramenta de apoio que pode ser uma efetiva estratégia de ensino e aprendizagem, quando desenvolvida e utilizada à luz de abordagens pedagógicas adequadas.

\section{CONCLUSÃo}

A hipermídia foi avaliada com boa e excelente usabilidade pelos discentes e docentes, respectivamente. A hipermídia, disponibilizada no link http://escoladesaude.ufrn.br/saeufrn/, pode ser utilizado pelas diversas instituições de ensino que formam profissionais de enfermagem dos diferentes níveis acadêmicos, bem como pelos serviços assistenciais, como um subsídio para a efetivação desse campo de saber. 
Como limitações, destaca-se a alta taxa de não resposta dos discentes ao formulário eletrônico. Sugere-se a utilização de outra estratégia de coleta de dados para investigadores que pretendam incluir este público.

Espera-se contribuir com a discussão acerca da necessidade de se avaliar os recursos tecnológicos educacionais, desenvolvidos a partir de abordagens pedagógicas e metodológicas apropriadas. Sem este processo, tem-se o risco de utilizar materiais de ensino carentes de objetivos educacionais adequados.

\section{REFERÊNCIAS}

AGAMA-SARABIA, A.; TREJO-NIÑO, G.; DE-LA-PEÑA-LEÓN, B.; ISLAS-ORTEGA, M.; CRESPO-KNOPFLER, S.; MARTÍNEZ-FELIPE, L.; GONZÁLEZ-VELÁZQUEZ, M.S. Audiovisual Aids in nursing education: literature review. Enfermería Global, v. 47, p. 512-525, 2017.

ÁLVAREZ-NIETO, C.; RICHARDSON, J.; PARRA-ANGUITA, G.; LINARES-ABAD, M.; HUSS, N.; GRANDE-GASCÓN, M.L.; GROSE, J.; HUYNEN, M.; LÓPEZ-MEDINA, I.M. Developing digital educational materials for nursing and sustainability: The results of an observational study. Nurse Education Today, v. 60, p. 139-146, 2018.

ASSOCIAÇÃO BRASILEIRA DE NORMAS TÉCNICAS (ABNT). ISO 9241 (Parte 11): Orientações sobre Usabilidade. ABNT, 2011.

AUSUBEL, D.P. Adquisición y retención del conocimiento: una perspectiva cognitiva. Barcelona: Ediciones Paidós Ibérica, 2002.

BANGOR, A.; KORTUM, P.; MILLER, J. Determining What Individual SUS Scores Mean: Adding an Adjective Rating Scale. Journal of Usability Studies, v. 4, n. 3, p. 114-123, 2009.

BRAGA, C.S.R.; ANDRADE, E.M.L.R.; LUZ, M.H.B.A.; MONTEIRO, A.K.C.; CAMPOS, M.O.O.B;; SILVA, F.M.S.; GONÇALVES NETA, F.C.C.; SANTOS, A.M.R.; PEREIRA, A.F.M. Construction and validation of a virtual learning object on intestinal elimination ostomy. Investigación y Educación en Enfermería, v. 34, n. 1, p.120-127, 2016.

BRASIL. Conselho Federal de Enfermagem. Pesquisa Perfil da Enfermagem no Brasil: Banco de Dados. Brasília, 2016.

BUTTON, D.; HARRINGTON, A.; BELAN, I. E-learning \& information communication technology (ICT) in nursing education: a review of the literature. Nurse Education Today, v. 34, n. 10, p. 1311-1323, 2014.

COCCE, A.L.R.; SILVEIRA, L.M.; GOÉS, F.S.N.; SOUZA, A.L.T.; STABILE, A.M. O ensino da anatomia nas escolas de enfermagem: um estudo descritivo. Arquivos de Ciências da Saúde, v. 24, n. 4, p. 8-13, 2017.

FROTA, N.M.; GALINDO NETO, N.H.; BARROS, L.M.; PEREIRA, F.G.F.; MELO, G.A.A.; CAETANO, J.A. Hypermedia on peripheral venipuncture: effectiveness in teaching nursing students. Revista Brasileira de Enfermagem, v. 71, n. 6, p. 2983-2989, 2018.

GARCÍA-BARRERA, A. Evaluación de recursos tecnológicos didácticos mediante e-rúbricas. Revista de Educación a Distancia, v. 49, n. 13, p. 1-12, 2016. 
GÓES, F.S.N.; FONSECA, L.M.M.; CAMARGO, R.A.A.; OLIVEIRA, G.F.; FELIPE, H.R. Educational technology "Anatomy and Vital Signs": Evaluation study of content, appearance and usability. International Journal of Medical Informatics, v. 84, n. 11, p. 982-987, 2015.

HORTENSE, F.T.P.; BERGEROT, C.D.; DE DOMENICO, E.B.L. Construction and validation of clinical contents for development of learning objects. Revista Brasileira de Enfermagem, v. 71, n. 2, p. 306-313, 2018.

PASQUALI, L. Instrumentação psicológica: fundamentos e práticas. Porto Alegre: Artmed, 2010.

PEREIRA, F.G.F.; SILVA, D.V.; SOUSA, L.M.O.; FROTA, N.M. Building a digital application for teaching vital signs. Revista Gaúcha de Enfermagem, v. 37, n. 2, e59015, 2016.

SALVADOR, P.T.C.O.; BEZERRIL, M.S.; MARIZ, C.M.S.; FERNANDES, M.I.D.; MARTINS, J.C.A.; SANTOS, V.E.P. Virtual learning object and environment: a concept analysis. Revista Brasileira de Enfermagem, v. 70, n. 3, p. 572-579, 2017.

SALVADOR, P.T.C.O.; MARIZ, C.M.S.; VÍTOR, A.F.; FERREIRA JÚNIOR, M.A.; FERNANDES, M.I.D.; MARTINS, J.C.A.; SANTOS, V.E.P. Validation of virtual learning object to support the teaching of nursing care systematization. Revista Brasileira de Enfermagem, v. 71, n. 1, p. 11-19, 2018.

SOUSA, C.S.; TURRINI, L.R.T.; POVEDA, F.B. Translation and adaptation of the instrument "suitability assessment of materials" (sam) into portuguese. Journal of Nursing UFPE on line, v. 9, n. 5, p. 7854-7861, 2015.

SPERONI, K.S.; VIZZOTTI, J.K. Recursos midiáticos na educação a distância em enfermagem: uma revisão narrativa da literatura. Vidya, v. 35, n. 1, p. 41-56, 2015.

TEIXEIRA, E.; MOTA, V.M.S.S. (organizadoras). Tecnologias educacionais em foco. São Paulo: Difusão, 2011.

\section{(cc) $\mathrm{EY}$}

Este trabalho está licenciado com uma Licença Creative Commons - Atribuição 4.0 Internacional. 